\title{
1 An ensemble prediction model for COVID-19 mortality risk
}

3

4

5

6
${ }^{1}$ School of Computer Science and Technology, Harbin Institute of Technology, China

${ }^{2}$ Department of Medical Genetics, University of Calgary, Canada

${ }^{3}$ Department of Medicine, McGill University, Canada

*Corresponding author: J.L \& E. W, jieli@hit.edu.cn, edwin.wang@ucalgary.ca, \#Co-first author

\section{Abstract}

Background: It's critical to identify COVID-19 patients with a higher death risk at early stage to give them better hospitalization or intensive care. However, thus far, none of the machine learning models has been shown to be successful in an independent cohort. We aim to develop a machine learning model which could accurately predict death risk of COVID-19 patients at an early stage in other independent cohorts.

Methods: We used a cohort containing 4711 patients whose clinical features associated with patient physiological conditions or lab test data associated with inflammation, hepatorenal function, cardiovascular function and so on to identify key features. To do so, we first developed a novel data preprocessing approach to clean up clinical features and then developed an ensemble machine learning method to identify key features.

Results: Finally, we identified 14 key clinical features whose combination reached a good predictive 

independent cohort containing 15,790 patients.

Conclusions: Our study shows that 14 key features are robust and useful in predicting the risk of death to help in making clinical decisions.

Keywords: COVID-19, SARS-CoV-2, Mortality prediction, Prognosis, Cohort studies

\section{Background}

The global COVID-19 pandemic is putting high pressure on healthcare systems around the world

[1-3]. Most of people infected with the SARS-CoV-2 have mild disease and self-limiting, but there

still a significant proportion of patients developed severe disease or even death $[4,5]$. In epidemic

areas, the shortage of medical resources may lead to an increase in mortality [6]. Therefore, it is important to distinguish patients at high risk of severe illness or mortality from others in the early stages of disease development.

There have been several researchers contributing to the areas of mortality risk prediction for patients. In May 2020, Yan et al. selected three biomarkers that predict the mortality of individual patients more than ten days in advance through machine learning tools [7]. However, Yan et al. gathered samples from a cohort of only 485 patients with confirmed SARS-CoV-2 infection may not be sufficient, and their mortality predictive model may not perform well in other cohorts [8]. Comorbidity, biomarkers for kidney disease and other clinical characteristics have also been associated with the severity of a patient's disease, according to previous studies [9-12]. Liang et al. proposed a deep learning-based survival model can predict the risk of COVID-19 patients 
44

this study is that the features selected may not sufficient to reflect the patient's condition, which might be the reason for the differences in the performance of different validation sets [13]. Based on clinical information, Altschul et al. proposed a novel severity score to assess the severity of patients infected with the SARS-CoV-2 [14]. Patients were classified into low (0-3), moderate (4-6), and high (7-10) COVID-19 severity scores. A ROC curve analysis showed that the AUC of the derivation cohort was 0.824 and the AUC of the validation cohort was 0.798 [14].

Except these examples, thus far, lots of similar efforts have been made by others. Recently, Wynants et al. conducted a comprehensive and systematic review of 145 prediction models from 107 studies, with a brief summary of the features (predictors) used by these models [15]. The key message from this analysis was that none of the models can be validated independently (i.e., their predictions failed when validating in an independent cohort), in another word, none of the predictive models developed in the COVID-19 domain could be used in clinics for decision marking. The prediction power of most of the models was similar to that of flipping a coin [15]. In this study, we look forward to developing a machine learning model which could accurately predict mortality risk of COVID-19 patients at an early stage in other independent cohorts.

\section{Methods and Materials}

The flow chart of our prediction method is shown in Figure 1. We first group the features and preprocess them, including processing extreme values and imputing missing values. Then, feature selection is carried out for constructing ensemble model (EM). Five base models: Gradient Boosted Decision Tree (GBDT), Extreme Gradient Boosting (XGBoost) [16], Random Forest (RF), Logistic Regression (LR), and Support Vector Machine (SVM) are used to select features. Features selected by more than half ( 3 or more) of the base models are used to construct the the 
medRxiv preprint doi: https://doi.org/10.1101/2022.01.10.22268985; this version posted January 13, 2022. The copyright holder for this preprint (which was not certified by peer review) is the author/funder, who has granted medRxiv a license to display the preprint in perpetuity.

It is made available under a CC-BY-NC-ND 4.0 International license .

66

67

68

69

70

EM. Finally, performance of the EM and base models is compared and validated on independent datasets.

\section{Data sets}

Two datasets are used in the study: Cohort 1 and Cohort 2. Cohort 1 with 4,711 COVID-19 patients $(1,148$ deaths) is from a recent study [14], which was collected from March 1, 2020, to April 16, 2020. All patients in cohort 1 are hospitalized, and their clinical features are obtained at admission[14]. Clinical features include patient's age, mean arterial pressure, oxygen saturation, etc., and the details and statistical information of these clinical features are shown in Table 1 and Supplementary Table 1. According to the types and meanings of clinical features (Table 1), numerical variables (features) that can directly reflect the physiological conditions of patients are selected features of models. These clinical features include age, oxygen saturation (OsSats), temperature (Temp), mean arterial pressure (MAP), D-dimer (Ddimer), platelets (Plts), international normalized ratio (INR), blood urea nitrogen (BUN), creatinine, sodium, glucose, aspartate aminotransferase (AST), alanine aminotransferase (ALT), white blood cells (WBC), lymphocytes (Lympho), interleukin-6 (IL-6), ferritin, C-reactive protein (CrctProtein), procalcitonin and troponin. If COVID-19 patient dies, his or her label is set to 1, otherwise it will be set to 0 . We select features and trained our models on Cohort 1 . Cohort 2 is an independent validation data containing 15,790 COVID-19 patients from UK biobank $[17,18]$. The statistical results of clinical features and population structure of this data are shown in Supplementary Table 2. The mortality rate is $4.21 \%(664 / 15790)$ in Cohort 2 . We selected hundreds of features (which are identical to or functionally related to the features selected in Cohort 1) from Cohort 2, and divided them into 55 functionally related features (Supplementary Table 3). These features 
included age, blood pressure-related features (such as hypertension), kidney function related features (such as creatinine), inflammation related features (such as monocyte), and so on.

\section{Feature grouping and feature preprocessing}

The presence of missing/error data will reduce the performance of the predictive model. Therefore, we developed a novel feature grouping and preprocessing method to deal with missing/error data.

We hypothesized that features closely related to patient's physiological conditions are better indicators associated with death risk. Thus, a key concept in this study is to select features and group them based on if they could indicate a particular aspect of the patient's physiology, but not strictly based on medical definitions. By doing so, age, OsSats, Temp, and MAP were divided into independent feature groups. Independent features are those that are not significantly associated each other. Other clinical features such as BUN, creatinine, glucose, sodium, ferritin, aspartate aminotransferase (AST), alanine aminotransferase (ALT) could indicate the conditions of liver or kidney [19-23], therefore, they were divided into the hepatorenal group. By the same token, IL-6, CrctProtein, Lympho, WBC, and procalcitonin are all inflammation-related features [24, 25], we combined these features into the inflammatory group. Troponin, AST, ALT, Ddimer, Plts, and INR could be associated with cardiovascular conditions [26-29], which were combined into the cardiovascular group. The detail of feature grouping is shown in Table 1.

One of the challenges of using clinical data is that clinical data often have missing values and error values (missing values are represented by 0 in the original dataset). To overcome this shortage, we developed a novel imputation pipeline to preprocess these features. First of all, we dealt with the extremums of some features. For each feature, we calculated its 95th percentile as the cut-off value (called cutoff95) and replaced feature value using cutoff95 if the feature value of 

the selected cutoff95 are shown in Table 2.

112 For the independent feature group, the missing values and obvious error values (temperature $50{ }^{\circ} \mathrm{C}$ and $-17.78^{\circ} \mathrm{C}$ ) were imputed with the mean value of the feature. We take the temperature feature as an example to illustrate how to impute the missing temperature value. First, dataset was divided into two groups (death and survival groups) according to whether the patient died or not, the mean temperatures of the two groups of patients were calculated, respectively, after removing 0 and obvious error values (the mean temperature of death group was defined as M1, the mean temperature of survival group was defined as M2). Then, we imputed the missing and error values within the group using the mean of each group (M1 for the death group, M2 for the survival 120 group).

121 For other feature groups (i.e., inflammatory group, etc.), the missing values were imputed using

122 k-nearest neighbor method $(\mathrm{KNN}, \mathrm{k}=3)$. We take WBC in inflammatory group as an example to 123 illustrate how to impute the missing WBC value. First, patients were divided into two groups 124 (death and survival groups) according to whether the patient died or not. Then, patients in the same group were clustered using KNN according to 4 features: Lympho, IL6, CrctProtein,

Procalcitonin. If WBC value of certain patient is missing, it is imputed by average WBC value of cardiovascular conditions, but they are more associated with hepatorenal features.

\section{Base model parameter settings}

131 In order to select valuable features and develop an EM which could take advantages of several 
132

133

134

135

136

137

base models such as Gradient Boosted Decision Tree (GBDT), Extreme Gradient Boosting (XGBoost) [16], Random Forest (RF), Logistic Regression (LR), and Support Vector Machine (SVM), we first conducted experiments in Cohort 1 and set the best parameters for five base models, respectively. For RF, GBDT, and XGBoost, we adjusted the number of decision trees (n_estimators), and for XGBoost, we also adjusted the maximum depth (max_depth) and the subsample ratio of features (colsample_bytree) to control over-fitting or under-fitting when constructing each tree. For the SVM model, we chose the radial basis function (RBF) (kernel) as the kernel function, and the regularization parameter $\mathrm{C}(\mathrm{C})$ was set to 0.7 to reduce overfitting. Since SVM favors the majority class on unbalanced datasets, we adjusted the weights of the two classes inversely proportional to the frequency of the classes (class_weight) in the dataset. In addition, z-score was used to standardize the data before input into LR and SVM models due to the characteristics of the algorithm. The detail of parameters of five base models is shown in Table 3.

\section{Feature selection for ensemble model}

Redundant features could be detrimental to predictive models to make correct predictions.

Therefore, we screened out most valuable features from the clinical features to improve the performance of our predictive models. The feature selection process is divided into two steps. In the first step, we select high performance feature set for 5 base models, respectively, from the 20 features in Table 1. In the second step, we combine feature sets of five base models to form the final selected feature set.

Genetic algorithm (GA) is used to select feature set [30], which is a heuristic search algorithm that simulates the process of natural selection. In the feature selection process, the area under the ROC 
154

155

156

157

158

curve (AUC) of each base model is taken as the objective function, each individual in GA represents a set of features, consisting of a binary string called a chromosome, and multiple individuals constitute a population. In each generation, a subset of individuals with the highest fitness (maximizing the objective function) goes into the next generation. In this way, we finally select a feature set that makes the predictive model get the best performance.

Taking the feature selection for GBDT as an example, the population size is set to 40 , and each chromosome is encoded into a binary string of length 20. Each position of the chromosome represents whether the corresponding feature is selected or not. We used the elite-tournament method[31] as a selection operator to select the chromosomes with the highest fitness in the population. The single-point crossover operator is chose as the offspring chromosome recombination method, the crossover probability is set to 0.7 and the probability of offspring mutation is the reciprocal of chromosome length. According to the above settings, after running 200 generations, the high performance feature set is selected for GBDT.

We also select high performance feature set for other base models. Thus each base model has a high performance feature set. We combine these feature sets, and select features that appear in more than half ( 3 or more) of the feature sets to form the final selected feature set.

\section{Ensemble model construction}

In order to construct an EM, which could take advantages of the prediction results of several models, we chose the above five models as base models to construct our EM. Similar to the feature selection method mentioned above, we also used GA to find a set of coefficients $\mathrm{C}$ as the weight of the prediction results of the five base models. The prediction results of EM ( prob $_{\mathrm{em}}$ ) for patients are the weighted average of the prediction results of each base model $\left(\right.$ prob $\left._{\mathrm{i}}\right)$, as defined 
below:

$$
\operatorname{prob}_{e m}=\frac{\sum c_{i} * \operatorname{prob}_{i}}{\sum c_{i}}
$$

177 We used 0.5 as the threshold, and patient whose prediction result is higher than the threshold is

178 predicted as dead. To obtain this set of coefficients $\mathrm{C}$ using the GA, fitness score is calculated

179 using the AUC of the EM. We code each set of coefficients for five base models as a binary string (i.e., a chromosome), which length is 70. The number of individuals (chromosomes) in the population is set to 40 . Other parameters of GA, such as selection operators and crossover operators, are the same as those used in feature selection process.

\section{Performance evaluation of predictive models}

In this study, we evaluate the prediction performance of different models using accuracy, area under ROC curve (AUC), precision and recall. The definitions of accuracy, precision and recall are as followings:

$$
\begin{gathered}
\text { Accuracy }=\frac{T P+T N}{T P+F P+T N+F N} \\
\text { Precision }=\frac{T P}{T P+F P} \\
\text { Recall }=\frac{T P}{T P+F N}
\end{gathered}
$$

Here, TP, FP, TN, and FN represent the number of true positive, false positive, true negative and false negative, respectively. In this study, the death patient is positive sample. The predictive model gives the probability of death for each patient, and we set a threshold of 0.5 , above which the patient is considered to be dead. Using clinical data composed of selected features as inputs and patient status (alive or death) as labels, we performed half-half cross-validations 100 times for each model (including EM and base models) on the entire cohort (Cohort 1, n=4,711). Specifically, we first randomly chose half of the samples (2,355 patients) as training set and the other half 
194

195

196

197

198

199

(2,356 patients) as test set from Cohort 1 . Then we trained EM and base models on training set and tested the performance of these models on test set. Subsequently, training set and test set was exchanged (that is, the former training set was the test set and the former test set was the training set), and these models were retrained and tested. At the same time, prediction results of these models were saved. Above process repeats 100 times, average values and standard deviations (SD) of accuracy, AUC, precision and recall of EM and five base models were calculated.

\section{Validation on independent dataset}

Cohort 2 is an independent cohort containing 15,790 patients (664 patients died and 15,126 patients survived). Since Cohort 2 did not fully contain the features in Cohort 1, we selected 55 features in Cohort 2 that were identical or functionally related to features selected from Cohort 1 (as shown in Supplementary Table 3 in this study) for further analysis. The missing data in Cohort 2 is imputed according to the method used in Cohort 1 . In order to validate the performance of different models in Cohort 2, we first used GA to select a feature set that was most related to the mortality risk of patients from 55 features. Cohort 2 and Cohort 1 are different in several aspects, such as mortality rate, age distribution and so on (Supplementary Figure 1, Supplementary Tables 1 and 2). For comparison purposes, next, we selected a subset of patients aged 50 to 84 years from Cohort 1 (Subset 1), since the patients in Cohort 2 ranged in age from 50-84 years. As with Cohort 1, the data in subset 1 was also half-and-half cross-validation for 100 times. Subsequently, we randomly sampled a subset of patients (subset 2) with a similar percentage of patients surviving in subset 1 from Cohort 2 and run a half-and-half cross-validation on subset 2. The process also repeats 100 times. Finally, we test different models on subset 1 and subset 2 and calculated their accuracy, AUC, precision and recall. 


\section{Results}

We first selected feature set for each base model in Cohort 1 according our method, respectively.

Feature sets of different base model were shown in Supplementary Table 4. Then, 14 key features were chose as the final feature set, which are Age, OsSats, MAP, Ddimer, Glucose, WBC, Lympho, IL6, CrctProtein, Procalcitonin, Troponin, Plts, INR and ALT. Finally, EM was constructed and the coefficients of base models of the EM calculated by GA were 0.39620338 (GBDT), 0.9574559 (XGBoost), 0.26222304 (RF), 0.0315571 (LR), and 0.24549838 (SVM), respectively. Mean values and standard deviations (SD) of accuracy, AUC, precision and recall of EM and five base models were shown in Table 4. Experimental results indicated that feature preprocessing and selection significantly improved the performance of the predictive models. In addition, the EM reached the best performance in unprocessed and preprocessed data, which showed the robustness of the EM. We also conducted an experiment in Cohort 1 using different imputation methods and test the performance of different models. Experimental results on Cohort 1 suggested that KNN imputation method was best and improved the performance of the predictive models than simply replacing missing data with 0 in the original data (as shown in Supplementary Table 5).

We calculated the mean value of the prediction results of the EM for each patient in 100 rounds to study the changes in precision and recall of EM when the threshold changed from 0 to 1 (Figure 2). With the increasing of the threshold, the precision had a trend of rapidly increasing at first and then slowly increasing, and correspondingly, the recall had a trend of slowly decreasing at first and then rapidly declining. Our goal was to find a reasonable range of thresholds in which the precision and recall can have a practical value. We selected the threshold $(0.24)$ when the recall reached 0.8 and the threshold $(0.46)$ when the precision reached 0.8 , and marked it with a dashed 

respectively.

model.

We further explored whether a single clinical feature could be used to stratify patients for mortality risk. To do so, we selected the first six clinical features which have a higher importance. $\mathrm{mg} / \mathrm{dL}$, patients have higher death risk.

\section{Experimental results on an independent dataset}

255 Finally, we further validate our predictive models on an independent cohort (Cohort 2). First, we 
performs best in three of the four performance metrics, which indicates that EM has a good robustness.

\section{Comparison with COVID-19 severity scores}

Altschul et al. collected COVID-19 patients' clinical information (Cohort 1) and also developed a compare EM and the CSS. In CSS, patients were classified into low risk (0-3 points), moderate according to the average prediction probability of each patient. The comparison results are shown patients who died to the high-risk group. These results indicated that feature preprocessing, feature selection and EM are helpful in improving predictive performance.

\section{Discussion}

In this study, we developed a novel data preprocessing method to deal with complex clinical data, and an EM to predict high-risk COVID-19 patients. Most importantly, we trained and tested the models in a large cohort and successfully validated the predictive models in a large independent cohort. This is the first study to show that high-risk COVID-19 predictive models and key features are able to reproducible for the predictions in a large independent cohort, suggesting that they could be potentially useful in clinical settings.

By comparing of our model with COVID-19 severity scores [14], we showed that our missing clinical features imputation method and the EM more accurately help physicians predict patients' 
282

283

284

285

286

287

mortality risk. In addition, we used GA to select the most appropriate 14 features from the 20 clinical features, and demonstrated that a removing of redundant features significantly improved the performance of the predictive models. The feature importance analysis showed that mean arterial pressure (MAP), interleukin-6 (IL-6), procalcitonin, D-dimer (Ddimer), age, and glucose were the most important features affecting the mortality risk of patients.

We used GA to find the optimal combination coefficient of comprehensive usage of five predictive models to construct the EM. In 100 rounds of half-half cross-validation, the EM achieved the best performance in multiple evaluation indicators. Moreover, we analyzed the precision and recall of each model under different thresholds to help clinicians in making choices according to the availability of clinical information. If physiological indicators, especially clinical features that reflect inflammation, hepatorenal function, and cardiovascular function can be obtained during the patient's stay in hospital, our models could be easily used to predict high-risk patients timely.

Our study showed that clinical features, such as age, MAP, and features that are associated with physiological status of the patient, can contribute to the predictive model of mortality stratification for COVID-19 in patients. The physiological status of coagulation function (related feature: Ddimer), hepatorenal function (related feature: glucose), and cardiac function (related feature: troponin) also had a noteworthy effect on mortality, which is consistent with previous findings [9-12]. In addition, we provided reference ranges for clinical features to help physicians quickly stratify patients using our models.

The experiments on the Cohort 2 demonstrated the correctness of our feature selection and the robustness of the predictive model. Despite the differences in population characteristics such as age distribution, ethnic proportion between cohort 1 and Cohort 2 (Supplementary Tables 1 and 2), 
304

305

and the inconsistent clinical features adopted (Supplementary Table 3), our prediction method still achieved good performance. These results further confirm that age, MAP, and clinical features related to inflammation, coagulation, hepatorenal function, and cardiovascular function can be used to predict the risk of death in patients with COVID-19.

Finally, we compared our model with those published by others [7, 13, 14, 32-35] (Supplementary

Table 7). We first compared features of different models. Overall, age, features associated with inflammation, kidney function, cardiovascular function, and lung function were selected for multiple studies, suggesting that the features we selected were more reasonable. Moreover, we employ more efficient feature selection methods to improve model prediction performance. Then, we compare the frameworks used by different studies. Three studies adopted the gradient boosting framework [7, 33, 34], another three adopted the deep learning framework [13, 32, 35], and one invented a scoring method [14]. Our model (EM) takes advantage of the gradient boosting frameworks (XGBoost and GBDT) with proven predictive performance, as well as the random forest model, logistic regression model, and support vector machine. Finally, we compared performance of different models. Three of the models were not completely consistent with our model in the selection of predicted clinical outcomes, such as severity of the disease[13, 35] and distinguishing COVID-19 patients from other pneumonia patients[32]. Furthermore, our model still achieved a good performance from the perspective of the comparison of the models' discriminative ability. For the remaining models, our model had the best discriminative performance compared with Rechtman et al.'s model [33] and the COVID-19 severity score[14].

Compared to Barda et al. 's study[34] (Supplementary Table 8), our subjects had a higher percentage of deaths and the AUC of our model was slightly lower than their model, but we 
achieved a higher precision when we achieved the same recall. Compared with the research of Yan et al.[7], their 'single-tree XGBoost' model has an outstanding predictive performance (AUC: 0.9506), but they chose only three features and their study cohort consisted of only 485 patients, making their model unreliable and not performing well on the tests of others [8]. In general, our predictive model (EM) is effective in predicting COVID-19 mortality risk.

There are also some limitations in this study. First of all, for cohort 1 (the training set), the patient population we studied was mainly hospitalized patients, and they generally exhibited more severe symptoms and therefore had a higher mortality rate than the general population, which may have caused some bias in our predictive model in the general population. Second, the characteristics of the cohort may vary performance of models and its ability to be validated. For example, the model's performance was slightly lower in cohort 2 than cohort 1 , because the structure of the two cohorts, such as age distribution, sex ratio, mortality rate, etc. is different. In addition, although we adopt functionally similar features, the differences between these features may also be responsible for the difference in model performance between cohorts. Moreover, since most of the clinical features adopted in this study were missing to varying degrees, the imputed data were affected by other data, which may affect the accuracy of the predictive model. Finally, COVID-19 pandemics are often accompanied by surges in patient numbers, resulting in difficulties in collecting all the required clinical features data, which will limit the application of our predictive model.

\section{Conclusions}

In summary, we selected 14 clinical features from 20 clinical features, and comprehensively utilized five predictive models to construct our predictive model: the EM, which had the best 
348

performance on multiple predictive evaluation indicators for COVID-19 mortality risk. Most importantly, EM was successfully validated in an independent cohort containing a large number of patients. We also studied the changes of precision and recall of each model under different thresholds, so as to provide reference for doctors to select appropriate thresholds according to medical resources. In addition, feature importance analysis showed that clinical features related to inflammation, hepatorenal function, and cardiovascular function were good predictors for COVID-19 mortality risk, which was consistent with previous studies.

\section{List of Abbreviations}

OsSats: oxygen saturation; Temp: temperature; MAP: mean arterial pressure; Ddimer: D-dimer; Plts: platelets; INR: international normalized ratio; BUN: blood urea nitrogen; AST: aspartate aminotransferase; ALT: alanine aminotransferase; WBC: white blood cells; Lympho: lymphocytes; IL-6: interleukin-6; CrctProtein: C-reactive protein; KNN: k-nearest neighbor method; GBDT: Gradient Boosted Decision Tree, XGBoost: Extreme Gradient Boosting; RF: Random Forest; LR: Logistic Regression; SVM: Support Vector Machine; EM: Ensemble Model; ROC: Receiver Operating Characteristic; AUC: Area Under ROC Curve; TP: True Positive; FP: False Positive, TN: True Negative; FN: False Negative; CSS: COVID-19 severity scores.

\section{Declarations}

\section{Ethics approval and consent to participate}

Informed consent was waived due to the nature of study being retrospective.

\section{Consent for publication}

Not applicable

\section{Availability of Data and materials}


corresponding author. The source code for data analysis is available.

There are no conflicts of interests for all authors.

\section{$374 \quad$ Funding}

375 Alberta Innovates for Health

\section{Authors' contributions}

377 JL and EW were responsible for the conception and design of the study. YW provided support. JL,

\section{Acknowledgements}

Not applicable

\section{Reference}

1. Arabi YM, Murthy S, Webb S. COVID-19: a novel coronavirus and a novel challenge for critical care. Intensive Care Medicine. 2020;46(5):833-6.

2. Grasselli G, Pesenti A, Cecconi M. Critical Care Utilization for the COVID-19 Outbreak in Lombardy, Italy: Early Experience and Forecast During an Emergency Response. JAMA. 2020;323(16):1545-6. 3. Xie J, Tong Z, Guan X, Du B, Qiu H, Slutsky AS. Critical care crisis and some recommendations during the COVID-19 epidemic in China. Intensive Care Medicine. 2020;46(5):837-40.

391 4. Guan W-j, Ni Z-y, Hu Y, Liang W-h, Ou C-q, He J-x, et al. Clinical Characteristics of Coronavirus Disease 2019 in China. New England Journal of Medicine. 2020;382(18):1708-20.

393 5. Wu Z, McGoogan JM. Characteristics of and Important Lessons From the Coronavirus Disease 3942019 (COVID-19) Outbreak in China: Summary of a Report of 72]314 Cases From the Chinese Center 395 for Disease Control and Prevention. JAMA. 2020;323(13):1239-42.

396 6. Ji Y, Ma Z, Peppelenbosch MP, Pan Q. Potential association between COVID-19 mortality and 397 health-care resource availability. The Lancet Global Health. 2020;8(4):e480.

398 7. Yan L, Zhang H-T, Goncalves J, Xiao Y, Wang M, Guo Y, et al. An interpretable mortality prediction 
model for COVID-19 patients. Nature Machine Intelligence. 2020;2(5):283-8.

8. Barish M, Bolourani $S$, Lau LF, Shah $S$, Zanos TP. External validation demonstrates limited clinical utility of the interpretable mortality prediction model for patients with COVID-19. Nature Machine Intelligence. 2021;3(1):25-7.

9. Yang $X, Y u Y, X U J$, Shu $H, X i a$ Ja, Liu $H$, et al. Clinical course and outcomes of critically ill patients with SARS-CoV-2 pneumonia in Wuhan, China: a single-centered, retrospective, observational study. The Lancet Respiratory Medicine. 2020;8(5):475-81.

10. Richardson S, Hirsch JS, Narasimhan M, Crawford JM, McGinn T, Davidson KW, et al. Presenting Characteristics, Comorbidities, and Outcomes Among 5700 Patients Hospitalized With COVID-19 in the New York City Area. JAMA. 2020;323(20):2052-9.

11. Cheng $Y$, Luo $R$, Wang $K$, Zhang $M$, Wang $Z$, Dong $L$, et al. Kidney disease is associated with in-hospital death of patients with COVID-19. Kidney International. 2020;97(5):829-38.

12. Wu S, Du Z, Shen S, Zhang B, Yang H, Li X, et al. Identification and Validation of a Novel Clinical Signature to Predict the Prognosis in Confirmed Coronavirus Disease 2019 Patients. Clinical Infectious Diseases. 2020;71(12):3154-62.

13. Liang W, Yao J, Chen A, Lv Q, Zanin M, Liu J, et al. Early triage of critically ill COVID-19 patients using deep learning. Nature Communications. 2020;11(1):3543.

14. Altschul DJ, Unda SR, Benton J, de la Garza Ramos R, Cezayirli P, Mehler M, et al. A novel severity score to predict inpatient mortality in COVID-19 patients. Scientific Reports. 2020;10(1):16726.

15. Wynants L, Van Calster B, Collins GS, Riley RD, Heinze G, Schuit E, et al. Prediction models for diagnosis and prognosis of covid-19: systematic review and critical appraisal. BMJ. 2020;369:m1328.

16. Chen T, Guestrin C. XGBoost: A Scalable Tree Boosting System. Proceedings of the 22nd ACM SIGKDD International Conference on Knowledge Discovery and Data Mining; San Francisco, California, USA: Association for Computing Machinery; 2016. p. 785-94.

17. Sudlow C, Gallacher J, Allen N, Beral V, Burton P, Danesh J, et al. UK biobank: an open access resource for identifying the causes of a wide range of complex diseases of middle and old age. PLoS Med. 2015;12(3):e1001779.

18. Barbour V. UK Biobank: a project in search of a protocol? The Lancet. 2003;361(9370):1734-8.

19. Nath KA, Grande JP, Farrugia G, Croatt AJ, Belcher JD, Hebbel RP, et al. Age sensitizes the kidney to heme protein-induced acute kidney injury. American Journal of Physiology-Renal Physiology. 2013;304(3):F317-F25.

20. Ybarra J, Fernández S, Sánchez-Hernández J, Romeo JH, Ballesta-Lopez C, Guell J, et al. Serum alanine aminotransferase predicts interventricular septum thickness and left ventricular mass in patients with nonalcoholic fatty liver disease. European Journal of Gastroenterology \& Hepatology. 2014;26(6):654-60.

21. Palekar NA, Naus R, Larson SP, Ward J, Harrison SA. Clinical model for distinguishing nonalcoholic steatohepatitis from simple steatosis in patients with nonalcoholic fatty liver disease. Liver International. 2006;26(2):151-6.

22. Cano N. Bench-to-bedside review: Glucose production from the kidney. Critical Care. 2002;6(4):317.

23. McNabb WR, Noormohamed FH, Lant AF. The Effects of Enalapril on Blood Pressure and the Kidney in Normotensive Subjects under Altered Sodium Balance. Journal of Hypertension. 1986;4(1):39-47.

24. Frasca D, Blomberg BB. Inflammaging decreases adaptive and innate immune responses in mice 
443 and humans. Biogerontology. 2016;17(1):7-19.

444 25. Galetto-Lacour A, Zamora SA, Gervaix A. Bedside Procalcitonin and C-Reactive Protein Tests in

445 Children With Fever Without Localizing Signs of Infection Seen in a Referral Center. Pediatrics.

446 2003;112(5):1054.

447 26. Kennergren C, Mantovani V, Lönnroth P, Nyström B, Berglin E, Hamberger A. Monitoring of 448 Extracellular Aspartate Aminotransferase and Troponin $\mathrm{T}$ by Microdialysis during and after 449 Cardioplegic Heart Arrest. Cardiology. 1999;92(3):162-70.

450 27. Schindhelm RK, Dekker JM, Nijpels G, Bouter LM, Stehouwer CDA, Heine RJ, et al. Alanine 451 aminotransferase predicts coronary heart disease events: A 10-year follow-up of the Hoorn Study. 452 Atherosclerosis. 2007;191(2):391-6.

453 28. Verni CC, Davila A, Jr, Sims CA, Diamond SL. D-Dimer and Fibrin Degradation Products Impair 454 Platelet Signaling: Plasma D-Dimer Is a Predictor and Mediator of Platelet Dysfunction During Trauma. 455 The Journal of Applied Laboratory Medicine. 2020;5(6):1253-64.

456 29. Ellis RJ, Mayo MS, Bodensteiner DM. Ciprofloxacin-warfarin coagulopathy: A case series. 457 American Journal of Hematology. 2000;63(1):28-31.

458 30. Yang J, Honavar V. Feature Subset Selection Using a Genetic Algorithm. In: Liu H, Motoda H, 459 editors. Feature Extraction, Construction and Selection: A Data Mining Perspective. Boston, MA: 460 Springer US; 1998. p. 117-36.

461 31. Blickle T, Thiele L. A Comparison of Selection Schemes Used in Evolutionary Algorithms. 462 Evolutionary Computation. 1996;4(4):361-94.

463 32. Wang S, Zha Y, Li W, Wu Q, Li X, Niu M, et al. A fully automatic deep learning system for COVID-19 464 diagnostic and prognostic analysis. European Respiratory Journal. 2020;56(2):2000775.

465 33. Rechtman E, Curtin P, Navarro E, Nirenberg S, Horton MK. Vital signs assessed in initial clinical 466 encounters predict COVID-19 mortality in an NYC hospital system. Scientific Reports. 467 2020;10(1):21545.

468 34. Barda N, Riesel D, Akriv A, Levy J, Finkel U, Yona G, et al. Developing a COVID-19 mortality risk 469 prediction model when individual-level data are not available. Nature Communications. 470 2020;11(1):4439.

471 35. Ning W, Lei S, Yang J, Cao Y, Jiang P, Yang Q, et al. Open resource of clinical data from patients 472 with pneumonia for the prediction of COVID-19 outcomes via deep learning. Nature Biomedical 473 Engineering. 2020;4(12):1197-207. 
medRxiv preprint doi: https://doi.org/10.1101/2022.01.10.22268985; this version posted January 13, 2022. The copyright holder for this preprint (which was not certified by peer review) is the author/funder, who has granted medRxiv a license to display the preprint in perpetuity.

\section{It is made available under a CC-BY-NC-ND 4.0 International license .}

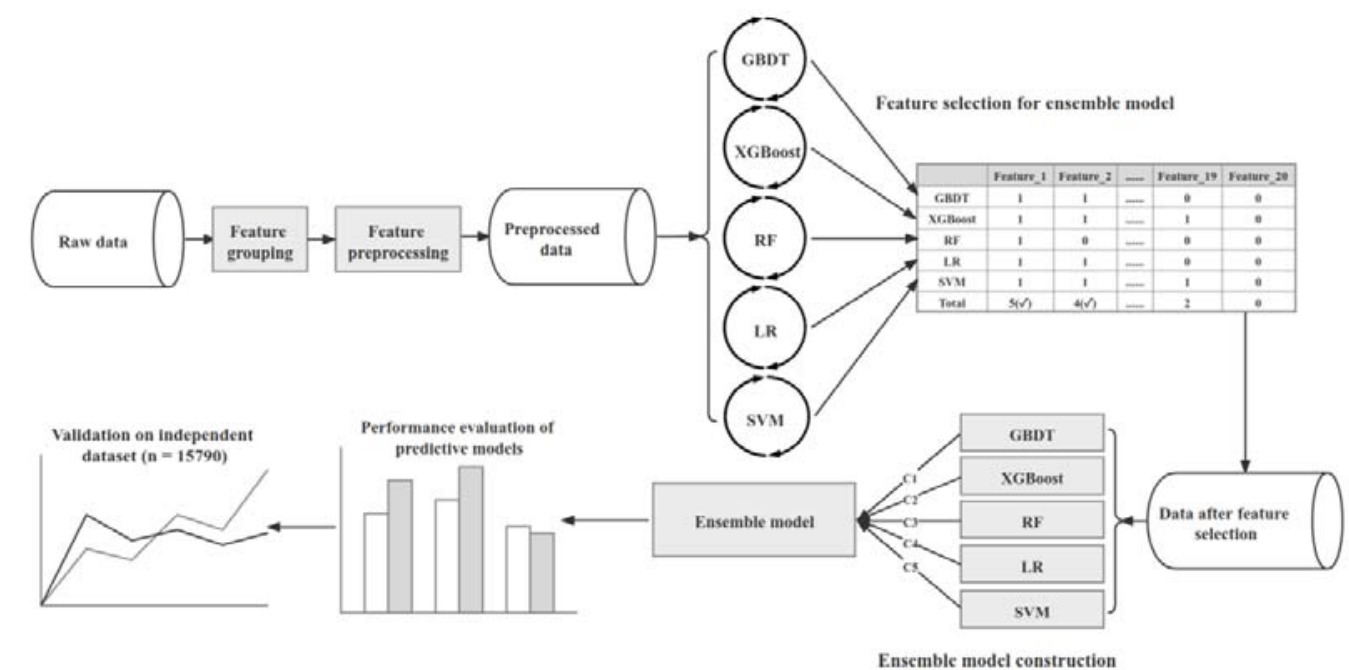

475

Figure 1. The flow chart of our prediction method

477 
478

479

480

481

482

\section{Ensemble Model}

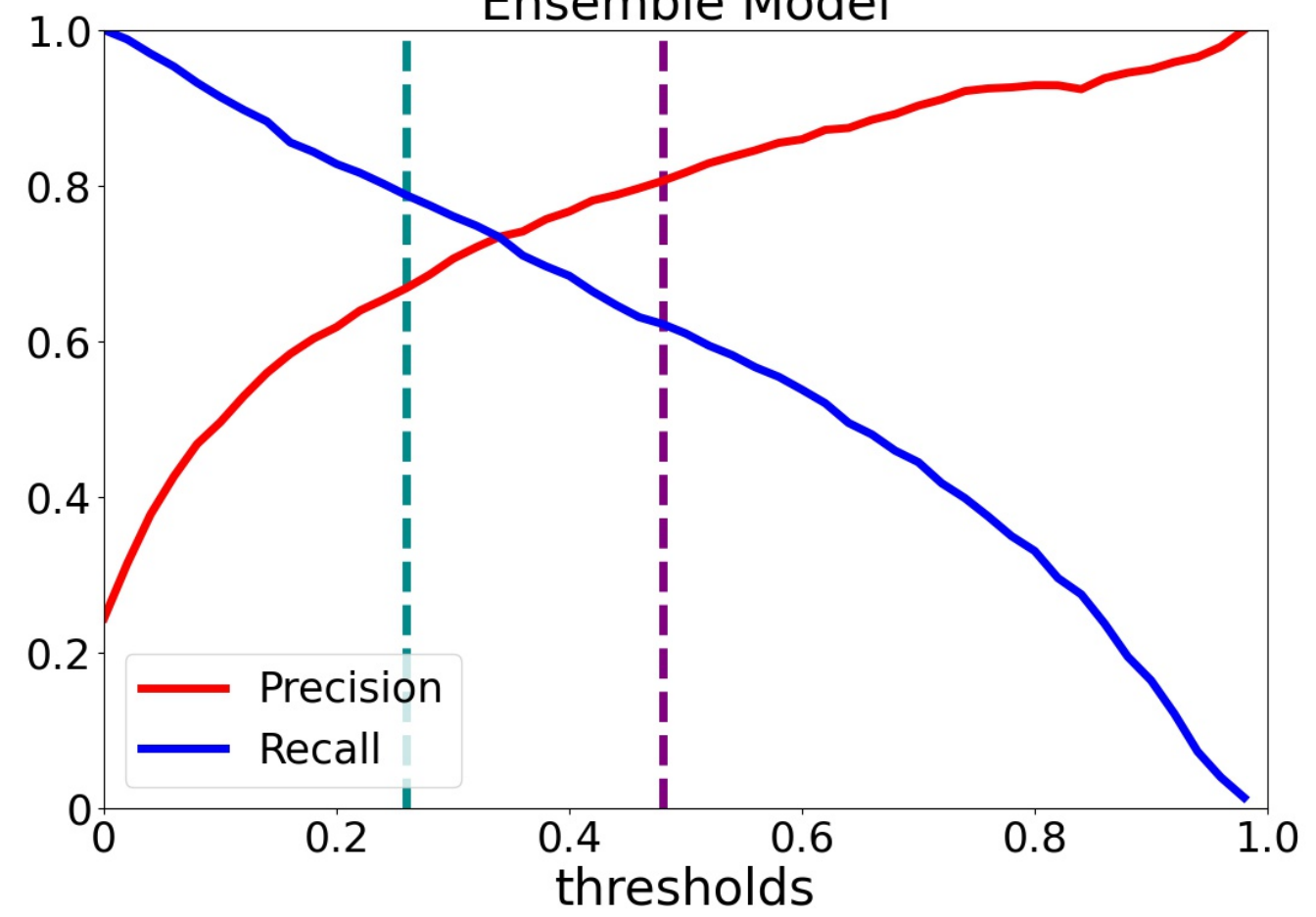

Figure 2. Changes in precision and recall of ensemble model under different thresholds. The $\mathrm{x}$ axis represents the threshold, and the y axis represents the value of precision and recall. Precision and recall are represented by red curve and blue curve respectively. The threshold when the precision is 0.8 and the threshold when the recall is 0.8 are indicated by the dashed purple line and the dashed cyan line respectively. 
medRxiv preprint doi: https://doi.org/10.1101/2022.01.10.22268985; this version posted January 13, 2022. The copyright holder for this preprint (which was not certified by peer review) is the author/funder, who has granted medRxiv a license to display the preprint in perpetuity.

\section{It is made available under a CC-BY-NC-ND 4.0 International license .}

483

484

485

486

487
Feature Importance

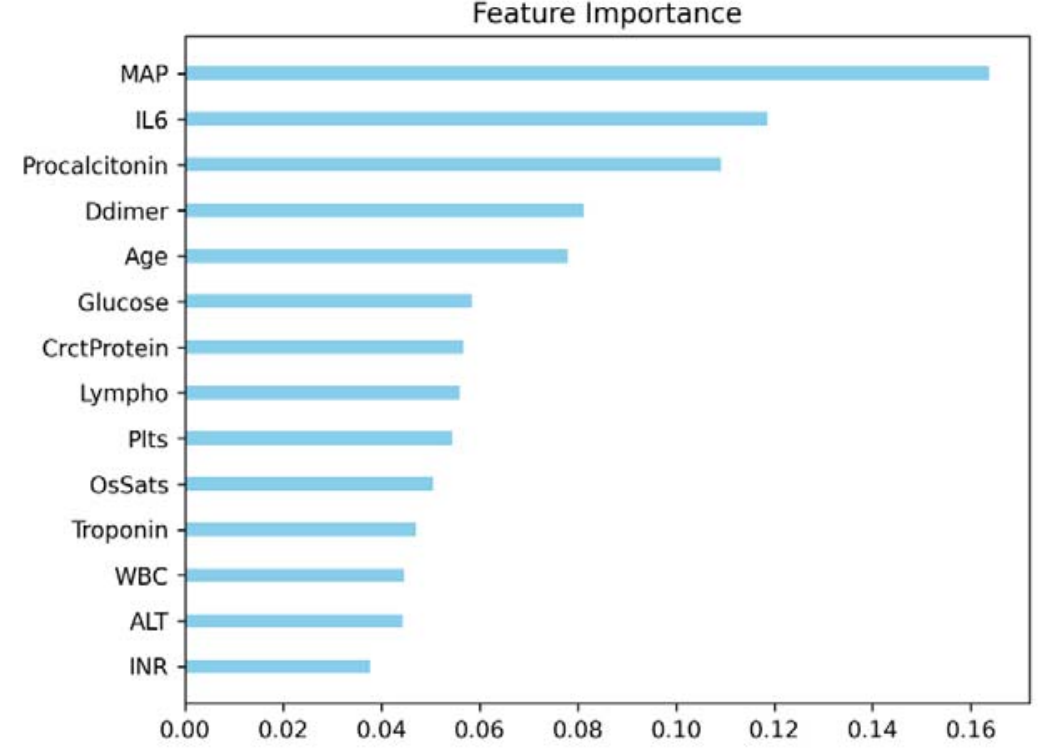

Figure 3. The mean of the importance of each feature in the XGBoost predictive model, over 100 rounds of predictions. 
medRxiv preprint doi: https://doi.org/10.1101/2022.01.10.22268985; this version posted January 13, 2022. The copyright holder for this preprint (which was not certified by peer review) is the author/funder, who has granted medRxiv a license to display the preprint in perpetuity.

It is made available under a CC-BY-NC-ND 4.0 International license .
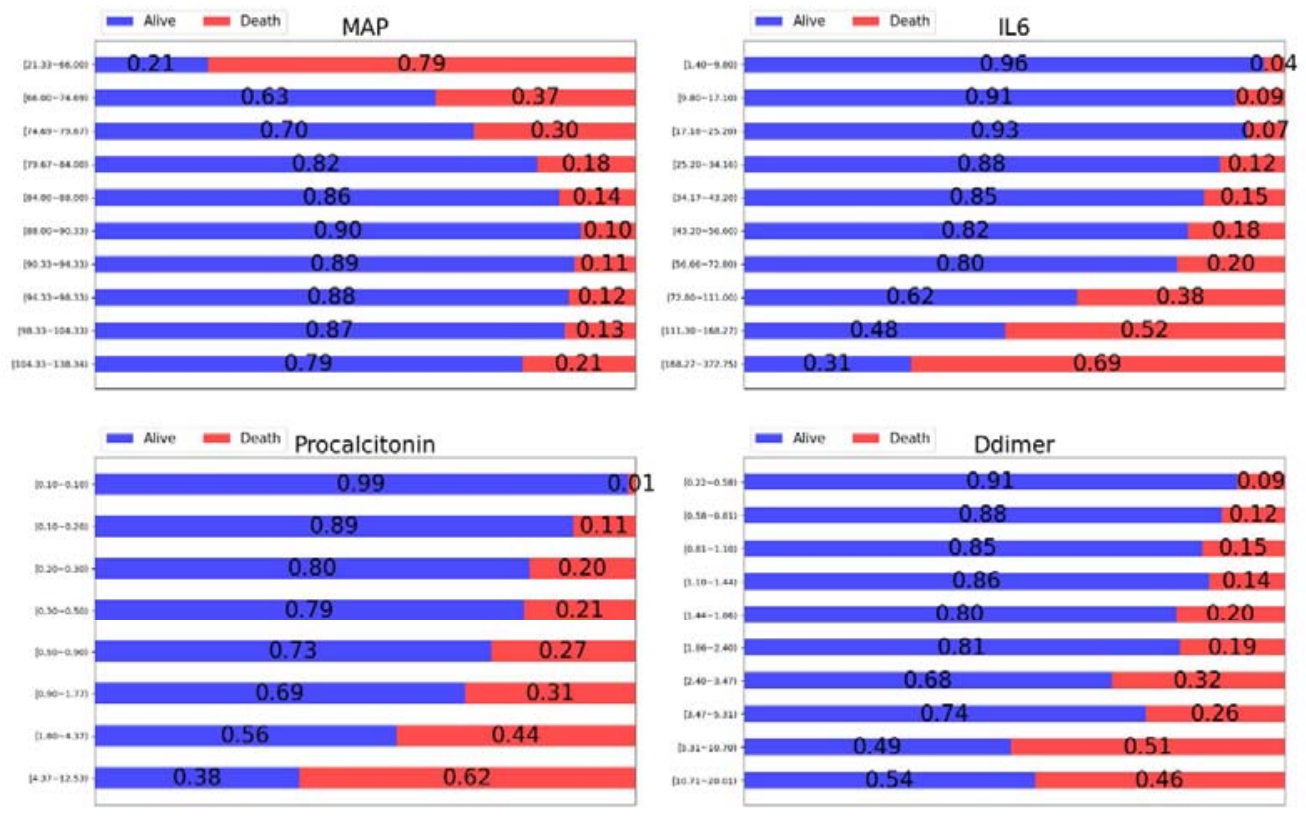

- Alive $=$ Death Age

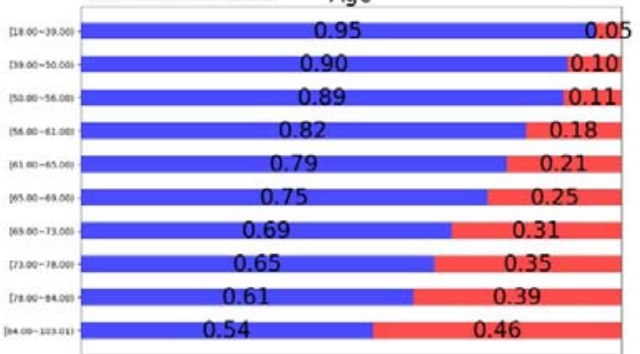

Alve Death Glucose

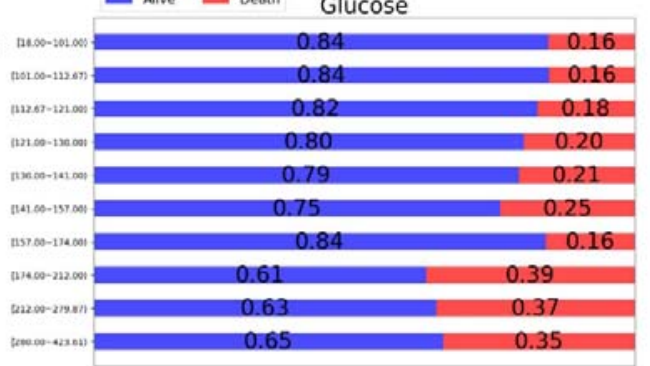

489 Figure 4. The proportion of patients alive or dead at different values for a single clinical feature. Patients who are

490 alive are shown in blue and those who are dead are shown in red, and the title of each subgraph represents the

491 clinical feature to which it belongs. 
Table 1. Clinical features of patients infected with SARS-CoV-2 in the cohort 1

\begin{tabular}{|c|c|c|c|c|}
\hline Features & $\begin{array}{l}\text { Description of } \\
\text { features }\end{array}$ & $\begin{array}{l}\text { Types of feature } \\
\text { attributes }\end{array}$ & Feature Groups & Impute method \\
\hline LOS & Length of hospital stay & Numerical variables & No group ${ }^{*}$ & Leave untreated \\
\hline Black & Race information & Binary variables & No group & Leave untreated \\
\hline White & Race information & Binary variables & No group & Leave untreated \\
\hline Asian & Race information & Binary variables & No group & Leave untreated \\
\hline Latino & Race information & Binary variables & No group & Leave untreated \\
\hline MI & Myocardial infraction & Binary variables & No group & Leave untreated \\
\hline PVD & $\begin{array}{l}\text { Peripheral vascular } \\
\text { disease }\end{array}$ & Binary variables & No group & Leave untreated \\
\hline $\mathrm{CHF}$ & Congestive heart failure & Binary variables & No group & Leave untreated \\
\hline CVD & cardiovascular disease & Binary variables & No group & Leave untreated \\
\hline DEMENT & dementia & Binary variables & No group & Leave untreated \\
\hline COPD & $\begin{array}{l}\text { Chronic obstructive } \\
\text { pulmonary disease }\end{array}$ & Binary variables & No group & Leave untreated \\
\hline $\begin{array}{l}\text { DM } \\
\text { Complicated }\end{array}$ & $\begin{array}{l}\text { diabetes mellitus } \\
\text { complicated }\end{array}$ & Binary variables & No group & Leave untreated \\
\hline DM Simple & diabetes mellitus simple & Binary variables & No group & Leave untreated \\
\hline Renal Disease & Renal Disease & Binary variables & No group & Leave untreated \\
\hline Stroke & Stroke & Binary variables & No group & Leave untreated \\
\hline Seizure & Seizure & Binary variables & No group & Leave untreated \\
\hline Age & Age & Numerical variables & $\begin{array}{l}\text { Independent } \\
\text { feature group } 1\end{array}$ & Leave untreated \\
\hline OsSats & Oxygen saturation & Numerical variables & $\begin{array}{l}\text { Independent } \\
\text { feature group } 2\end{array}$ & $\begin{array}{l}\text { Imputed by the } \\
\text { mean }\end{array}$ \\
\hline Temp & Temperature & Numerical variables & $\begin{array}{l}\text { Independent } \\
\text { feature group } 3\end{array}$ & $\begin{array}{l}\text { Imputed by the } \\
\text { mean }\end{array}$ \\
\hline MAP & Mean arterial pressure & Numerical variables & $\begin{array}{l}\text { Independent } \\
\text { feature group } 4\end{array}$ & $\begin{array}{l}\text { Imputed by the } \\
\text { mean }\end{array}$ \\
\hline Ddimer & D-dimer & Numerical variables & $\begin{array}{l}\text { Cardiovascular } \\
\text { group }\end{array}$ & $\begin{array}{l}\text { KNN imputing in } \\
\text { the same group }\end{array}$ \\
\hline Plts & Platelets & Numerical variables & $\begin{array}{l}\text { Cardiovascular } \\
\text { group }\end{array}$ & $\begin{array}{l}\text { KNN imputing in } \\
\text { the same group }\end{array}$ \\
\hline INR & $\begin{array}{l}\text { International } \\
\text { normalized ratio }\end{array}$ & Numerical variables & $\begin{array}{l}\text { Cardiovascular } \\
\text { group }\end{array}$ & $\begin{array}{l}\text { KNN imputing in } \\
\text { the same group }\end{array}$ \\
\hline Troponin & Troponin & Numerical variables & $\begin{array}{l}\text { Cardiovascular } \\
\text { group }\end{array}$ & $\begin{array}{l}\text { KNN imputing in } \\
\text { the same group }\end{array}$ \\
\hline BUN & Blood urea nitrogen & Numerical variables & $\begin{array}{l}\text { Hepatorenal } \\
\text { group }\end{array}$ & $\begin{array}{l}\text { KNN imputing in } \\
\text { the same group }\end{array}$ \\
\hline Creatinine & Creatinine & Numerical variables & $\begin{array}{l}\text { Hepatorenal } \\
\text { group }\end{array}$ & $\begin{array}{l}\text { KNN imputing in } \\
\text { the same group }\end{array}$ \\
\hline Sodium & Sodium & Numerical variables & Hepatorenal & KNN imputing in \\
\hline
\end{tabular}


medRxiv preprint doi: https://doi.org/10.1101/2022.01.10.22268985; this version posted January 13, 2022. The copyright holder for this preprint (which was not certified by peer review) is the author/funder, who has granted medRxiv a license to display the preprint in perpetuity.

It is made available under a CC-BY-NC-ND 4.0 International license .

\begin{tabular}{|c|c|c|c|c|}
\hline & & & group & the same group \\
\hline Glucose & Glucose & Numerical variables & $\begin{array}{l}\text { Hepatorenal } \\
\text { group }\end{array}$ & $\begin{array}{l}\text { KNN imputing in } \\
\text { the same group }\end{array}$ \\
\hline Ferritin & Ferritin & Numerical variables & $\begin{array}{l}\text { Hepatorenal } \\
\text { group }\end{array}$ & $\begin{array}{l}\text { KNN imputing in } \\
\text { the same group }\end{array}$ \\
\hline AST & $\begin{array}{l}\text { Aspartate } \\
\text { aminotransferase }\end{array}$ & Numerical variables & $\begin{array}{l}\text { Hepatorenal } \\
\text { group \& } \\
\text { Cardiovascular } \\
\text { group }\end{array}$ & $\begin{array}{l}\text { KNN imputing in } \\
\text { the same group }\end{array}$ \\
\hline ALT & $\begin{array}{l}\text { Alanine } \\
\text { aminotransferase }\end{array}$ & Numerical variables & $\begin{array}{l}\text { Hepatorenal } \\
\text { group \& } \\
\text { Cardiovascular } \\
\text { group }\end{array}$ & $\begin{array}{l}\text { KNN imputing in } \\
\text { the same group }\end{array}$ \\
\hline WBC & While blood cells & Numerical variables & $\begin{array}{l}\text { Inflammatory } \\
\text { group }\end{array}$ & $\begin{array}{l}\mathrm{KNN} \text { imputing in } \\
\text { the same group }\end{array}$ \\
\hline Lympho & Lymphocytes & Numerical variables & $\begin{array}{l}\text { Inflammatory } \\
\text { group }\end{array}$ & $\begin{array}{l}\text { KNN imputing in } \\
\text { the same group }\end{array}$ \\
\hline IL6 & Interleukin-6 & Numerical variables & $\begin{array}{l}\text { Inflammatory } \\
\text { group }\end{array}$ & $\begin{array}{l}\mathrm{KNN} \text { imputing in } \\
\text { the same group }\end{array}$ \\
\hline CrctProtein & C-reactive protein & Numerical variables & $\begin{array}{l}\text { Inflammatory } \\
\text { group }\end{array}$ & $\begin{array}{l}\text { KNN imputing in } \\
\text { the same group }\end{array}$ \\
\hline Procalcitonin & Procalcitonin & Numerical variables & $\begin{array}{l}\text { Inflammatory } \\
\text { group }\end{array}$ & $\begin{array}{l}\mathrm{KNN} \text { imputing in } \\
\text { the same group }\end{array}$ \\
\hline All CNS & No introduction found & Binary variables & No group & Leave untreated \\
\hline Pure CNS & No introduction found & Binary variables & No group & Leave untreated \\
\hline OldSyncope & No introduction found & Binary variables & No group & Leave untreated \\
\hline OldOtherNeuro & No introduction found & Binary variables & No group & Leave untreated \\
\hline OtherBrnLsn & No introduction found & Binary variables & No group & Leave untreated \\
\hline $\begin{array}{l}\text { Derivation } \\
\text { cohort }\end{array}$ & $\begin{array}{l}\text { Grouping in the original } \\
\text { literature [14] }\end{array}$ & Binary variables & No group & Leave untreated \\
\hline Death & $\begin{array}{l}\text { Whether the patient } \\
\text { died or not }\end{array}$ & Binary variables & No group & Leave untreated \\
\hline Severity & $\begin{array}{l}\text { COVID-19 Severity } \\
\text { (Score given in the } \\
\text { original literature) }[14]\end{array}$ & Binary variables & No group & Leave untreated \\
\hline
\end{tabular}

*: No group means that the feature is not used in this study.

Table 2. The maximum in the original data and the selected cutoff95

\begin{tabular}{|l|l|l|}
\hline Feature & Maximum & Cutoff95 \\
\hline Ddimer & 20.00001 & 20.00001 \\
\hline Plts & 1226 & 433 \\
\hline INR & 17.0001 & 1.7 \\
\hline
\end{tabular}




\begin{tabular}{|l|l|l|}
\hline BUN & 301 & 97 \\
\hline Creatinine & 31.66 & 7.35 \\
\hline Sodium & 170.001 & 153 \\
\hline Glucose & 1000.001 & 423.6 \\
\hline AST & 10000 & 159 \\
\hline ALT & 3228 & 116 \\
\hline WBC & 219.7 & 16.9 \\
\hline Lympho & 209.1 & 2.4 \\
\hline IL6 & 111040 & 372.74 \\
\hline Ferritin & 100000 & 4508.55 \\
\hline CrctProtein & 100.0001 & 34.3 \\
\hline Procalcitonin & 50.0001 & 12.52 \\
\hline Troponin & 9.56 & 0.21 \\
\hline
\end{tabular}

Table 3. Parameter settings of five base models

\begin{tabular}{|l|l|l|l|l|}
\hline GBDT & XGBoost & RF & LR & SVM \\
\hline random_state $=$ & $\begin{array}{l}\text { random_state }=10 \\
\text { learning_rate }=0.1 \\
\text { n_estimators }=150 \\
\text { learning_rate } \\
0.1\end{array}$ & random_state $=10$ & random_state=10 & random_state $=10$ \\
n_estimators $=$ & & & kernel='rbf' \\
110 & $\begin{array}{l}\text { max_depth }=12 \\
\text { colsample_bytree=0 } \\
\text { class_weight='bala } \\
\text { use_label_encoder= } \\
\text { False }\end{array}$ & & & nced' \\
& & & C=0.7 \\
probability=True
\end{tabular}

Table 4. Performance results of different models on Cohort 1

\begin{tabular}{|c|c|c|c|c|c|c|}
\hline $\begin{array}{l}\text { Unprocessed } \\
\text { data in Cohort } 1\end{array}$ & GBDT & XGBoost & $\mathrm{RF}$ & LR & SVM & EM \\
\hline Accuracy (SD) & $0.834(0.005)$ & $0.830(0.004)$ & $0.832(0.004)$ & $0.811(0.005)$ & $0.813(0.006)$ & $0.837(0.004)$ \\
\hline AUC (SD) & $0.847(0.007)$ & $0.844(0.007)$ & $0.848(0.007)$ & $0.803(0.007)$ & $0.826(0.007)$ & $0.854(0.006)$ \\
\hline Precision (SD) & $0.736(0.020)$ & $0.750(0.019)$ & $0.754(0.020)$ & $0.707(0.020)$ & $0.688(0.024)$ & $0.772(0.020)$ \\
\hline Recall (SD) & $0.495(0.020)$ & $0.454(0.018)$ & $0.460(0.020)$ & $0.385(0.019)$ & $0.424(0.021)$ & $0.471(0.019)$ \\
\hline $\begin{array}{l}\text { Data in Cohort } 1 \\
\text { after } \\
\text { preprocessing } \\
\text { and feature } \\
\text { selection }\end{array}$ & GBDT & XGBoost & $\mathrm{RF}$ & LR & SVM & EM \\
\hline Accuracy (SD) & $0.864(0.005)$ & $0.864(0.005)$ & $0.862(0.005)$ & $0.847(0.004)$ & $0.855(0.006)$ & $0.868(0.005)$ \\
\hline $\mathrm{AUC}(\mathrm{SD})$ & $0.900(0.005)$ & $0.904(0.005)$ & $0.900(0.005)$ & $0.870(0.006)$ & $0.890(0.005)$ & $0.907(0.005)$ \\
\hline Precision (SD) & $0.774(0.018)$ & $0.805(0.019)$ & $0.791(0.019)$ & $0.764(0.018)$ & $0.738(0.020)$ & $0.804(0.019)$ \\
\hline Recall (SD) & $0.625(0.016)$ & $0.582(0.017)$ & $0.588(0.017)$ & $0.542(0.019)$ & $0.628(0.021)$ & $0.605(0.016)$ \\
\hline
\end{tabular}


501 Table 5. The percentage of patients who survived or died in the low, moderate, or high-risk group

\begin{tabular}{|c|r|r|r|r|r|r|}
\hline \multirow{2}{*}{$\begin{array}{c}\text { Predictive } \\
\text { Model }\end{array}$} & \multicolumn{2}{|c|}{ The percentage of patients who survived } & \multicolumn{3}{|c|}{ The percentage of patients who died } \\
\cline { 2 - 7 } & Low risk & Moderate risk & High risk & Low risk & Moderate risk & \multicolumn{1}{c|}{ High risk } \\
\hline CSS & 88.25 & 60.98 & 22.03 & 11.75 & 39.02 & 77.97 \\
\hline EM & $\mathbf{9 0 . 1 5}$ & 40.09 & 9.73 & 9.85 & 59.91 & $\mathbf{9 0 . 2 7}$ \\
\hline
\end{tabular}

502

503

Table 6. Performance of different models on an independent dataset

\begin{tabular}{|l|l|l|l|l|l|l|}
\hline Subset 1 & GBDT & XGBoost & RF & LR & SVM & EM \\
\hline $\begin{array}{l}\text { Accuracy } \\
\text { (SD) }\end{array}$ & $0.850(0.006)$ & $0.849(0.006)$ & $0.850(0.006)$ & $0.828(0.006)$ & $0.838(0.006)$ & $0.854(0.005)$ \\
\hline AUC (SD) & $0.884(0.007)$ & $0.888(0.007)$ & $0.887(0.007)$ & $0.846(0.008)$ & $0.875(0.007)$ & $0.893(0.007)$ \\
\hline $\begin{array}{l}\text { Precision } \\
\text { (SD) }\end{array}$ & $0.764(0.020)$ & $0.797(0.021)$ & $0.788(0.021)$ & $0.749(0.020)$ & $0.723(0.021)$ & $0.799(0.020)$ \\
\hline Recall (SD) & $0.616(0.022)$ & $0.565(0.023)$ & $0.583(0.023)$ & $0.515(0.020)$ & $0.616(0.025)$ & $0.588(0.022)$ \\
\hline Subset 2 & GBDT & XGBoost & RF & LR & SVM & EM \\
\hline $\begin{array}{l}\text { Accuracy } \\
\text { (SD) }\end{array}$ & $0.800(0.009)$ & $0.804(0.009)$ & $0.803(0.009)$ & $0.804(0.009)$ & $0.786(0.009)$ & $0.810(0.009)$ \\
\hline AUC (SD) & $0.858(0.009)$ & $0.863(0.010)$ & $0.860(0.009)$ & $0.862(0.009)$ & $0.847(0.009)$ & $0.871(0.008)$ \\
\hline $\begin{array}{l}\text { Precision } \\
\text { (SD) }\end{array}$ & $0.644(0.023)$ & $0.687(0.029)$ & $0.662(0.025)$ & $0.654(0.024)$ & $0.612(0.024)$ & $0.684(0.026)$ \\
\hline Recall (SD) & $0.533(0.030)$ & $0.461(0.029)$ & $0.507(0.031)$ & $0.533(0.027)$ & $0.500(0.033)$ & $0.512(0.028)$ \\
\hline
\end{tabular}

504 